Washek F. Pfeffer*, Department of Mathematics, University of California, Davis, CA 95616, USA, e-mail: wfpfeffer@ucdavis.edu

\title{
THE GENERALIZED RIEMANN-STIELTJES INTEGRAL
}

\begin{abstract}
We define a generalized Riemann-Stieltjes integral with respect to charges (i.e., additive continuous functions on bounded $B V$ sets), and study the linear space of all functions that are integrable with respect to every charge.
\end{abstract}

Generalized Riemann-Stieltjes integrals with respect to charges (i.e., additive continuous functions on bounded $B V$ sets) arise naturally in studying multipliers for the generalized Riemann integral with respect to the Lebesgue measure (Proposition 2.9). Once around, they tend to make life on their own and generate interesting problems. The one of primary importance is to describe the linear space of all functions that are integrable with respect to every charge, or every charge in a given class. While this problem remains open in its full generality, some partial solutions can be found in the present paper. Specifically, we show the following.

- A function integrable with respect to all charges is essentially bounded and $B V$ (Proposition 3.1).

- Essentially bounded $B V$ functions which are pointwise Lipschitz are integrable with respect to all charges (Theorem 3.4).

- Simple $B V$ functions are integrable with respect to a broad class of charges (Proposition 3.6); in particular, they are multipliers for the generalized Riemann integral with respect to the Lebesgue measure.

\footnotetext{
Mathematical Reviews subject classification: Primary: 26B30, 26B20. Secondary: 26A39, 26A42

Received by the editors January 3, 1995

* Supported in part by a Fulbright grant while visiting the Center for Theoretical Study at Charles University in Prague, Czech Republic, and by the Catholic University of Louvain, Louvaine-la-Neuve, Belgium.
} 
Our main technique is based on the geometric idea introduced in [10]. To every charge $G$ in $\mathbb{R}^{m}$ we associate a unique charge $\hat{G}$ in $\mathbb{R}^{m+1}$ (Lemma 3.2). Given a nonnegative essentially bounded $B V$ function $f$ on $\mathbb{R}^{m}$, we show that $\hat{G}$ evaluated on the subgraphs of $f$ over bounded $B V$ sets defines a charge $F$ in $\mathbb{R}^{m}$. The charge $F$ is a natural candidate for the indefinite integral of $f$ with respect to $G$, and we prove that under suitable hypothesis, $F$ indeed is such an indefinite integral.

The paper is organized as follows. After necessary preliminaries given in Section 1, we define the generalized Riemann-Stieltjes integral in Section 2 and establish some of its basic properties. Section 3, devoted to the study of integrable functions, contains the main results. In section 4 , we present a generalization $W_{\text {loc }}(\Omega)$ of the Sobolev space $W_{\text {loc }}^{1,1}(\Omega)$, and prove a divergence theorem (Proposition 4.4) for continuous vector fields in $W_{\text {loc }}\left(\Omega, \mathbb{R}^{m}\right)$.

I acknowledge with pleasure that during the preparation of this paper I largely benefited from discussions with Josef Král, Jan Malý, Ivan Netuka and Thierry De Pauw.

\section{Preliminaries}

For an integer $m \geq 1$, we denote by $\mathbb{R}^{m}$ the $m$-fold Cartesian product of the set $\mathbb{R}$ of all real numbers. In $\mathbb{R}^{m}$ we use exclusively the maximum norm $|\cdot|$. The usual inner product of $x, y \in \mathbb{R}^{m}$ is denoted by $x \cdot y$, and $\|x\|=\sqrt{x \cdot x}$. For an $x \in \mathbb{R}^{m}$ and $\varepsilon>0$, we let $B[x, \varepsilon]=\left\{y \in \mathbb{R}^{m}:|x-y| \leq \varepsilon\right\}$. The closure, interior, boundary, and diameter of a set $E \subset \mathbb{R}^{m}$ are denoted by $\mathrm{cl} E$, int $E$, $\partial E$, and $d(E)$, respectively. A cell is a compact nondegenerate subinterval of $\mathbb{R}^{m}$, and a figure is a finite (possibly empty) union of cells.

By $\lambda$ and $\mathcal{H}$ we denote, respectively, the Lebesgue and $(m-1)$-dimensional Hausdorff measures in $\mathbb{R}^{m}$. For a set $E \subset \mathbb{R}^{m}$, we often write $|E|$ instead of $\lambda(E)$. The terms "measurable" and "absolutely continuous" as well as the expressions "almost all" and "almost everywhere" always refer to the measure $\lambda$. A set $E \subset \mathbb{R}^{m}$ is called, respectively, negligible or thin according to whether its $\lambda$ measure is zero or its $\mathcal{H}$ measure is $\sigma$-finite.

For $E \subset \mathbb{R}^{m}$ and $0 \leq \alpha \leq 1$, let

$$
E(\alpha)=\left\{x \in \mathbb{R}^{m}: \lim _{\varepsilon \rightarrow 0+} \frac{|E \cap B[x, \varepsilon]|}{(2 \varepsilon)^{m}}=\alpha\right\} .
$$

The sets int ${ }^{*} E=E(1), \mathrm{cl}^{*} E=\mathbb{R}^{m}-E(0)$, and $\partial^{*} E=\mathbb{R}^{m}-[E(0) \cup E(1)]$ are called the essential interior, essential closure, and essential boundary of $E$, respectively. We say $E$ is nondispersed whenever $\mathrm{cl} E=\mathrm{cl}^{*} E$. 
Unless specified otherwise, all functions we consider are real-valued. If $f$ is a function defined on a set $E \subset \mathbb{R}^{m}$, the sets

$$
\text { null } f=\{x \in E: f(x)=0\} \quad \text { and } \quad \operatorname{supp} f=\operatorname{cl}(E-\operatorname{null} f)
$$

are called the null set of $f$ and support of $f$, respectively.

A $B V$ function is a function $f \in L^{1}\left(\mathbb{R}^{m} ; \lambda\right)$ such that the distributional derivative of $f$ is a vector-valued measure $D f$ in $\mathbb{R}^{m}$ whose variation $\|D f\|$ is finite [6, Chapter 5]; we let $\|f\|=\|D f\|\left(\mathbb{R}^{m}\right)$. A $B V$ set is a set $A \subset \mathbb{R}^{m}$ whose characteristic function $\chi_{A}$ is $B V$. By $\mathcal{B} V_{c}^{\infty}$ and $B V_{c}$ we denote the families of all essentially bounded $B V$ functions with compact support and all bounded $B V$ sets, respectively. Using the obvious identification, we view $B V_{c}$ as a subset of the linear space $\mathcal{B} \mathcal{V}_{c}^{\infty}$. A set $A \subset \mathbb{R}^{m}$ is locally $B V$ if $A \cap B$ is $B V$ for each $B \in B V_{c}$.

For each compact set $K \subset \mathbb{R}^{m}$ and $n=1,2, \ldots$, the family

$$
\mathcal{B V}(K, n)=\left\{f \in \mathcal{B V}_{c}^{\infty}: \operatorname{supp} f \subset K \text { and }\|f\|_{\infty}+\|f\| \leq n\right\}
$$

is a compact subset of $L^{1}\left(\mathbb{R}^{m} ; \lambda\right)\left[6\right.$, Section 5.2.3]. We give $\mathcal{B} V_{c}^{\infty}$ the largest locally convex topology $\tau$ for which the embeddings $\mathcal{B V}(K, n) \hookrightarrow \mathcal{B V}_{c}^{\infty}$ are continuous. It is easy to verify that $\tau$ is a complete Hausdorff topology which is sequential [5, Section 1.7] but not metrizable. A sequence $\left\{f_{n}\right\}$ in $\mathcal{B V}_{c}^{\infty}$ $\tau$-converges to zero if and only if $\lim \left\|f_{n}\right\|_{1}=0$ and there is a compact set $K \subset \mathbb{R}^{m}$ and an $\alpha>0$ such that $\operatorname{supp} f_{n} \subset K$ and $\left\|f_{n}\right\|_{\infty}+\left\|f_{n}\right\| \leq \alpha$ for $n=1,2, \ldots$. The family $B V_{c}$, viewed as a subset of $\mathcal{B} \mathcal{V}_{c}^{\infty}$, is closed in $\mathcal{B} \mathcal{V}_{c}^{\infty}$.

If $\Omega \subset \mathbb{R}^{m}$ is an open set and $A \subset \mathbb{R}^{m}$ is a locally $B V$ set, the linear spaces

$$
\begin{aligned}
& \mathcal{B V}_{c}^{\infty}(\Omega)=\left\{f \in \mathcal{B V}_{c}^{\infty}: \operatorname{supp} f \subset \Omega\right\} \\
& \mathcal{B} \mathcal{V}^{\infty}(A)=\left\{f \in \mathcal{B} \mathcal{V}_{c}^{\infty}: \mathbb{R}^{m}-\operatorname{null} f \subset A\right\}
\end{aligned}
$$

and the families

$$
\begin{aligned}
B V_{c}(\Omega) & =\left\{A \in B V_{c}: \mathrm{cl} A \subset \Omega\right\} \\
B V(A) & =\left\{B \in B V_{c}: B \subset A\right\}
\end{aligned}
$$

are given the relative topology from $\mathcal{B V}_{c}^{\infty}$. The linear space $C_{c}^{1}(\Omega)$ is dense in $\mathcal{B V}_{c}^{\infty}(\Omega)$ [6, Section 5.2], and the family of all figures contained in $\Omega$ is dense in $B V_{c}(\Omega)$ [3, Proposition 1.1]. In particular, $\mathcal{B} V_{c}^{\infty}(\Omega)$ and $B V_{c}(\Omega)$ are separable, and so are $\mathcal{B} \mathcal{V}^{\infty}(A)$ and $B V(A)$.

Let $A \in B V_{c}$. The perimeter and exterior normal of $A$ are denoted by $\|A\|$ and $\nu_{A}$, respectively. By definition $\|A\|=\left\|\chi_{A}\right\|$, and it follows from 
[6, Section 5.11, Theorem 1] that $\|A\|=\mathcal{H}\left(\partial^{*} A\right)$. The regularity of $A$ is the number

$$
r(A)= \begin{cases}\frac{|A|}{d(A)\|A\|} & \text { if } d(A)\|A\|>0, \\ 0 & \text { otherwise. }\end{cases}
$$

By the isoperimetric inequality, $[2 r(A)]^{m} \leq|A| /[d(A)]^{m}$. The set of all $x \in \mathbb{R}^{m}$ such that

$$
\lim _{\varepsilon \rightarrow 0+} \frac{\left\|D \chi_{A}\right\|(B[x, \varepsilon])}{(2 \varepsilon)^{m-1}}>0
$$

is called the critical boundary of $A$, denoted by $\partial^{c} A$. According to [22, Section 4], $\partial^{*} A \subset \partial^{c} A \subset \partial A$ and $\mathcal{H}\left(\partial^{c} A-\partial^{*} A\right)=0$. The sets $\operatorname{int}^{c} A=\mathrm{cl}^{*} A-\partial^{c} A$ and $\operatorname{cl}^{c} A=\mathrm{cl}^{*} A \cup \partial^{c} A$ are called the critical interior and critical closure of $A$, respectively.

A nonnegative function $\delta$ on a set $E \subset \mathbb{R}^{m}$ is called a gage on $E$ whenever its null set is thin. A partition is a collection (possibly empty) $P=\left\{\left(A_{1}, x_{1}\right)\right.$, $\left.\ldots,\left(A_{p}, x_{p}\right)\right\}$ such that $A_{1}, \ldots, A_{p}$ are disjoint sets from $B V_{c}$, and $x_{i} \in \mathrm{cl}^{*} A_{i}$ for $i=1, \ldots, p$. Given $\varepsilon>0, E \subset \mathbb{R}^{m}$, and a gage $\delta$ on $E$, we say that $P$ is

1. $\varepsilon$-regular if $r\left(A_{i}\right)>\varepsilon$ for $i=1, \ldots, p$;

2. in $E$ if $\bigcup_{i=1}^{p} A_{i} \subset E$;

3. anchored in $E$ if $\left\{x_{1}, \ldots, x_{p}\right\} \subset E$;

4. $\delta$-fine if it is anchored in $E$ and $d\left(A_{i}\right)<\delta\left(x_{i}\right)$ for $i=1, \ldots, p$.

We note that our concept of partition coincide with that of tight partition introduced in [11, Section 7].

A charge in an open set $\Omega \subset \mathbb{R}^{m}$ or in a locally $B V$ set $A \subset \mathbb{R}^{m}$ is an additive $\tau$-continuous function $F$ defined on the family $B V_{c}(\Omega)$ or $B V(A)$. Explicitly, $F$ satisfies the following two conditions.

Additivity: $F(A \cup B)=F(A)+F(B)$ for each pair of disjoint sets $A, B$ in $B V_{c}(\Omega)$ or $B V(A)$, respectively.

Continuity: Given $\varepsilon>0$ and a compact set $K \subset \Omega$ or a bounded $B V$ set $B \subset A$, there is an $\eta>0$ such that $|F(C)|<\varepsilon$ for each $B V$ set $C$ contained in $K$ or $B$, respectively, for which $\|C\|<1 / \varepsilon$ and $|C|<\eta$.

If $F$ is a charge in $\Omega$ or in $A$, then $F(C)=0$ for each negligible set $C$ in $B V_{c}(\Omega)$ or in $B V(A)$, respectively. Given a charge $F$ in a locally $B V$ set $A \subset \mathbb{R}^{m}$, we let $(F \mathrm{~L} A)(B)=F(A \cap B)$ for each $B \in B V_{c}$. Clearly, $F \mathrm{~L} A$ is a charge in $\mathbb{R}^{m}$, called the canonical extension of $F$. 
Example 1.1 Let $\Omega \subset \mathbb{R}^{m}$ be an open set and let $v: \Omega \rightarrow \mathbb{R}^{m}$ be a continuous vector field. If $G(A)=\int_{\partial^{*} A} v \cdot \nu_{A} d \mathcal{H}$ for each $A \in B V_{c}(\Omega)$, then $G$ is a charge in $\Omega$, called the flux of $v$ [11, Example 4.2].

The $\tau$-closure of a family $\mathcal{E} \subset B V_{c}$ is denoted by $\mathcal{E}^{-}$. A kernel of a set $A \in B V_{c}$ is a family $\mathcal{A} \subset B V(A)$ such that $A \in \mathcal{A}^{-}$. If $\mathcal{A}$ is a kernel of $A \in B V_{c}$ and $\mathcal{B}$ is a kernel of $B \in B V_{c}$, then

$$
\mathcal{A} \vee \mathcal{B}=\{X \cup Y: X \in \mathcal{A}, Y \in \mathcal{B}\}
$$

and

$$
\mathcal{A} \wedge \mathcal{B}=\{X \cap Y: X \in \mathcal{A}, Y \in \mathcal{B}\}
$$

are kernels of $A \cup B$ and $A \cap B$, respectively [11, Lemma 8.3]. For further properties of kernels we refer to [11, Section 8]. If $F$ is a charge in $A \in B V_{c}$ and $\mathcal{A}$ is a kernel of $A$, then $F(A)$ is uniquely determined by $F \uparrow \mathcal{A}$.

The next three lemmas have been established in [10, Proposition 1] and [11, Lemmas 7.2 and 8.3].

Lemma 1.2 An additive function $F$ in an open set $\Omega \subset \mathbb{R}^{m}$ or in a locally $B V$ set $A \subset \mathbb{R}^{m}$ is a charge if and only if the following condition is satisfied: given $\varepsilon>0$ and a compact set $K \subset \Omega$ or a bounded $B V$ set $B \subset A$, there is a $\theta>0$ such that $|F(C)|<\theta|C|+\varepsilon(\|C\|+1)$ for each $B V$ set $C$ contained in $K$ or $B$, respectively.

Lemma 1.3 Let $F$ be a charge in $A \in B V$, and let $\delta$ be a gage on $\operatorname{cl}^{*} A$. For each positive $\varepsilon<1 /(2 m)$ there is an $\varepsilon$-regular $\delta$-fine partition $P$ in $A$ with $|F(A-\bigcup P)|<\varepsilon$.

Lemma 1.4 For each kernel $\mathcal{A}$ of a set $A \in B V_{c}$, there is a countable kernel $\mathcal{A}^{\prime}$ of $A$ such that each $B^{\prime} \in \mathcal{A}^{\prime}$ is a nondispersed subset of a $B \in \mathcal{A}$.

\section{The Integral}

Definition 2.1 Let $G$ be a charge in $A \in B V_{c}$. A function $f$ defined on $\mathrm{cl}^{*} A$ is called integrable in $A$ with respect to $G$ if there is a kernel $\mathcal{A}$ of $A$ and a charge $F$ in $A$ satisfying the following condition: given $B \in \mathcal{A}$ and $\varepsilon>0$, we can find a gage $\delta$ on $\mathrm{cl}^{*} B$ so that $\sum_{i=1}^{p}\left|f\left(x_{i}\right) G\left(B_{i}\right)-F\left(B_{i}\right)\right|<\varepsilon$ for each $\varepsilon$-regular $\delta$-fine partition $\left\{\left(B_{1}, x_{1}\right), \ldots,\left(B_{p}, x_{p}\right)\right\}$ in $B$. 
The family of all integrable functions in $A$ with respect to $G$ is denoted by $\mathcal{R}(A ; G)$. When the trivial kernel $\mathcal{A}=\{A\}$ can be used in Definition 2.1, we say $f$ is simply integrable (abbreviated as $s$-integrable), and we denote by $\mathcal{R}_{s}(A ; G)$ the family of all simply integrable functions in $A$ with respect to $G$. If $f \in \mathcal{R}(A ; G)$, then there is only one charge $F$ in $A$ that satisfies the condition of Definition 2.1; it is called the indefinite integral of $f$ in $A$ with respect to $G$.

Indeed, let $F_{1}$ and $F_{2}$ be two such charges, and let $B \in B V(A)$. Since $\mathcal{B}=\{B \cap C: C \in \mathcal{A}\}$ is a kernel of $B$, it suffices to show that $F_{1}(B)=F_{2}(B)$ for each $B \in B V(A)$ contained in a $C \in \mathcal{A}$. To this end, choose a positive $\varepsilon<1 /(2 m)$ and for $k=1,2$, find gauges $\delta_{k}$ on $\mathrm{cl}^{*} C$ that correspond to $F_{k}$ and $\varepsilon$ according to Definition 2.1. Then $\delta=\min \left\{\delta_{1}, \delta_{2}\right\}$ is a gage on $\mathrm{cl}^{*} C$, and hence on $\mathrm{cl}^{*} B$. By Lemma 1.3 , there is an $\varepsilon$-regular $\delta$-fine partition $P=\left\{\left(B_{1}, x_{1}\right)\right.$, $\left.\ldots,\left(B_{p}, x_{p}\right)\right\}$ in $B$, and hence in $C$, with $\left|\left(F_{1}-F_{2}\right)\left(B-\bigcup_{i=1}^{p} B_{i}\right)\right|<\varepsilon$. We obtain

$$
\begin{aligned}
\left|F_{1}(B)-F_{2}(B)\right| \leq & \left(F_{1}-F_{2}\right)\left(B-\bigcup_{i=1}^{p} B_{i}\right)\left|+\sum_{i=1}^{p}\right| F_{1}\left(B_{i}\right)-f\left(x_{i}\right) G\left(B_{i}\right) \mid \\
& +\sum_{i=1}^{p}\left|f\left(x_{i}\right) G\left(B_{i}\right)-F_{2}\left(B_{i}\right)\right|<3 \varepsilon
\end{aligned}
$$

and $F_{1}(B)=F_{2}(B)$ by the arbitrariness of $\varepsilon$.

If $F$ is the indefinite integral of $f$ in $A$ with respect to $G$, we call the number $F(A)$ the integral of $f$ over $A$ with respect to $G$, denoted by $\int_{A} f d G$. In view of Proposition 2.6 below, this notation will cause no confusion. Using the same symbol for functions and their restrictions, we note that $F(B)=\int_{B} f d G$ for each $B \in B V(A)$; since $f \in \mathcal{R}(B ; G)$ and $F$ is the indefinite integral of $f$ in $B$ with respect to $G$. The map $(f, G) \mapsto \int_{A} f d G$ is bilinear in the obvious way.

A completely Riemannian definition of the integral $\int_{A} f d G$ can be found in $\left[13\right.$, Section 2]. If $G=\lambda$, the integral $\int_{A} f d G$ coincides with the continuous integral of [11, Section 9] modified in accordance with [11, Remark 5.2,4(a)]; $s$-integrability with respect to $G$ coincides with variational integrability of $[11$, Definition 5.1], again modified in accordance with [11, Remark 5.2,4(a)].

By the definition of a gage, neither the integrability of $f$ nor its indefinite integral depend on values $f$ takes on thin sets. However, the integral $\int_{A} f d G$ may depend on the values $f$ takes on negligible sets, in particular, on $\mathrm{cl}^{*} A-A$. The following example and proposition clarify this dependence. 
Example 2.2 Assume $m=1$. In this case, each integrable function is $s$ integrable [11, Corollary 9.12]. Let $C$ be the Cantor ternary set in $[0,1]$, and let $G$ be the Cantor function on $[0,1]$ viewed as a charge in $[0,1]$ (see $[11$, Example 5.3.11] and Example 1.1). The set $A=[0,1]-C$ belongs to $B V_{c}$ and $\mathrm{cl}^{*} A=[0,1]$. Let $\delta$ be a gage on $[0,1]$ such that $C \cap B[x, \delta(x)]=\emptyset$ for each $x \in A$. If $\left\{\left(A_{1}, x_{1}\right), \ldots,\left(A_{p}, x_{p}\right)\right\}$ is a $\delta$-fine partition in $[0,1]$, then

$$
\sum_{i=1}^{p} \chi_{A}\left(x_{i}\right) G\left(A_{i}\right)=0
$$

and so $\int_{A} \chi_{A} d G=\int_{0}^{1} \chi_{A} d G=0$. On the other hand,

$$
\int_{A} \chi_{[0,1]} d G=\int_{0}^{1} \chi_{[0,1]} d G=G(A)=1
$$

and yet $\chi_{A}$ and $\chi_{[0,1]}$ differ only on a negligible set $C=\mathrm{cl}^{*} A-A$.

Let $\Omega \subset \mathbb{R}^{m}$ be an open set, and let $F$ be a function defined on $B V_{c}(\Omega)$. If $E \subset \Omega$, we let

$$
V_{*} F(E)=\sup _{0<\varepsilon<\frac{1}{2 m}} \inf _{\delta} \sup _{P} \sum_{i=1}^{p}\left|F\left(A_{i}\right)\right|
$$

where $\delta$ runs through all gauges on $E$ such that $B[x, \delta(x)] \subset \Omega$ for each $x \in$ $E$, and $P$ runs through all $\varepsilon$-regular partitions $P=\left\{\left(A_{1}, x_{1}\right), \ldots,\left(A_{p}, x_{p}\right)\right\}$ anchored in $E$ that are $\delta$-fine. It is easy to verify that the extended real-valued function $V_{*} F: E \mapsto V_{*} F(E)$ is a metric measure in $\Omega$ (cf. [21, Theorem 3.7] and $\left[14\right.$, Section 3.2]). By [3, Lemma 4.6], the measure $V_{*} F$ is Borel regular [6, Section 1.1.1].

Remark 2.3 Let $F$ be an additive continuous function in $A \in B V_{c}$, and let $E \subset \mathrm{cl}^{*} A$. Then it follows from [3, Lemma 4.10] that

$$
V_{*}(F \mathrm{~L} A)(E)=\sup _{0<\varepsilon<\frac{1}{2 m}} \inf _{\delta} \sup _{P} \sum_{i=1}^{p}\left|F\left(A_{i}\right)\right|
$$

where $\delta$ runs through all gauges on $E$, and $P$ runs through all $\varepsilon$-regular partitions $P=\left\{\left(A_{1}, x_{1}\right), \ldots,\left(A_{p}, x_{p}\right)\right\}$ in $A$ anchored in $E$ that are $\delta$-fine.

Proposition 2.4 Let $G$ be a charge in $A \in B V_{c}$, and let $E \subset \mathrm{cl}^{*} A$. The following conditions are equivalent. 
1. There is a kernel $\mathcal{A}$ of $A$ such that $V_{*}(G \mathrm{~L} B)\left(E \cap \mathrm{cl}^{*} B\right)=0$ for each $B \in \mathcal{A}$.

2. If $f$ is a function on $\mathrm{cl}^{*} A$ such that $f(x)=0$ for each $x \in \mathrm{cl}^{*} A-E$, then $f \in \mathcal{R}(A ; G)$ and $\int_{B} f d G=0$ for each $B \in B V(A)$.

Proof. Assume the first condition is met, and let $f$ be a function on $\mathrm{cl}^{*} A$ such that $f(x)=0$ for each $x \in \mathrm{cl}^{*} A-E$. It suffices to show that $f \in \mathcal{R}(A ; G)$ and $\int_{A} f d G=0$. To this end, select a $B \in \mathcal{A}$, and let

$$
E_{n}=\left\{x \in E \cap \mathrm{cl}^{*} B: n-1 \leq|f(x)|<n\right\}
$$

for $n=1,2, \ldots$ Choose an $\varepsilon>0$, and find a gage $\delta_{n}$ on $E_{n}$ so that

$$
\sum_{i=1}^{p}\left|G\left(B_{i}\right)\right|<\frac{\varepsilon}{n 2^{n}}
$$

for each $\varepsilon$-regular partition $\left\{\left(B_{1}, x_{1}\right), \ldots,\left(B_{p}, x_{p}\right)\right\}$ in $B$ anchored in $E_{n}$ that is $\delta_{n}$-fine. Since $E$ is the disjoint union of the sets $E_{1}, E_{2}, \ldots$, we can define a gage $\delta$ on $\mathrm{cl}^{*} B$ by letting

$$
\delta(x)= \begin{cases}\delta_{n}(x) & \text { if } x \in E_{n}, n=1,2, \ldots, \\ 1 & \text { if } x \in \mathrm{cl}^{*} B-E .\end{cases}
$$

Now if $\left\{\left(B_{1}, x_{1}\right), \ldots,\left(B_{p}, x_{p}\right)\right\}$ is an $\varepsilon$-regular $\delta$-fine partition in $B$, then

$$
\sum_{i=1}^{p}\left|f\left(x_{i}\right) G\left(B_{i}\right)\right| \leq \sum_{n=1}^{\infty} n \sum_{x_{i} \in E_{n}}\left|G\left(B_{i}\right)\right|<\sum_{n=1}^{\infty} n \frac{\varepsilon}{n 2^{n}}=\varepsilon,
$$

and the desired conclusion follows. Conversely, suppose $\chi_{E} \in \mathcal{R}(A ; G)$ and $\int_{B} \chi_{E} d G=0$ for each $B \in B V(A)$. Then there is a kernel $\mathcal{A}$ of $A$ with the following property: given $B \in \mathcal{A}$ and a positive $\varepsilon<1 /(2 m)$, we can find a gage $\delta$ on $\mathrm{cl}^{*} B$ so that $\sum_{i=1}^{p}\left|\chi_{E}\left(x_{i}\right) G\left(B_{i}\right)\right|<\varepsilon$ for each $\varepsilon^{\prime}$-regular $\delta$-fine partition $\left\{\left(B_{1}, x_{1}\right), \ldots,\left(B_{p}, x_{p}\right)\right\}$ in $B$, where $\varepsilon^{\prime}=\varepsilon^{m+1}$. With no loss of generality, we may assume $\delta(x)=0$ for each $x \in \mathrm{cl}^{*} B-\operatorname{int}^{c} B$. Now on $\mathrm{cl}^{*} B$, there is a gage $\delta^{\prime} \leq \delta$ such that if $\left\{\left(C_{1}, z_{1}\right), \ldots,\left(C_{s}, z_{s}\right)\right\}$ is an $\varepsilon$-regular partition anchored in $E \cap \mathrm{cl}^{*} B$ that is $\delta^{\prime}$-fine, then $\left\{\left(B \cap C_{1}, z_{1}\right), \ldots,\left(B \cap C_{s}, z_{s}\right)\right\}$ is an $\varepsilon^{\prime}$-regular $\delta$-fine partition in $B$ [12, Lemma 1.2]. Thus we obtain

$$
\sum_{i=1}^{s}\left|(G \mathrm{~L} B)\left(C_{i}\right)\right|=\sum_{i=1}^{s}\left|\chi_{E}\left(z_{i}\right) G\left(B \cap C_{i}\right)\right|<\varepsilon,
$$

and $V_{*}(G \mathrm{~L} B)\left(E \cap \mathrm{cl}^{*} B\right) \leq \varepsilon$. The proposition follows from the arbitrariness of $\varepsilon$. 
Definition 2.5 A charge $G$ in $A \in B V_{c}$ is called regular whenever it satisfies the following condition: given a negligible set $E \subset \mathrm{cl}^{*} A$, we can find a kernel $\mathcal{A}$ of $A$ such that $V_{*}\left(G\llcorner B)\left(E \cap \mathrm{cl}^{*} B\right)=0\right.$ for each $B \in \mathcal{A}$.

Let $G$ be a regular charge in $A \in B V_{c}$, and let $f$ be a function defined on $\mathrm{cl}^{*} A$. According to Proposition 2.4, the integrability of $f$ with respect to $G$ and the value of $\int_{A} f d G$ do not depend on the values $f$ takes on negligible subsets of $\mathrm{cl}^{*} A$.

Proposition 2.6 Let $\mu$ be a Borel measure in $\mathbb{R}^{m}$ whose restriction to $B V_{c}$ is a charge $G$ in $\mathbb{R}^{m}$. Then $\mu$ is $\sigma$-finite and absolutely continuous, and $\mu(E)=$ $V_{*} G(E)$ for every measurable set $E \subset \mathbb{R}^{m}$. If $A \in B V_{c}$, then each function $f$ from $L^{1}\left(\mathrm{cl}^{*} A ; \mu\right)$ belongs to $\mathcal{R}_{s}(A ; \mu)$ and we have

$$
\int_{A} f d G=\int_{A} f d \mu
$$

where on the right is the Lebesgue integral with respect to the measure $\mu$.

Proof. Since $\mu$ extends a charge in $\mathbb{R}^{m}$, it is $\sigma$-finite and absolutely continuous. By [3, Lemma 4.4], we have $\mu(K)=V_{*} G(K)$ whenever $K \subset \mathbb{R}^{m}$ is a cell. As $V_{*} G$ vanishes on thin sets, $\mu(U)=V_{*}(U)$ for each open set $U \subset \mathbb{R}^{m}$, and the first claim follows from [16, Theorem 2.18].

Given $f \in L^{1}\left(\mathrm{cl}^{*} A ; \mu\right)$, the map $F: C \mapsto \int_{C} f d \mu$ is a charge in $A$. Choose an $\varepsilon>0$ and use the Vitali-Carathéodory theorem [16, Theorem 2.25] to find extended real-valued functions $g$ and $h$ on $\mathrm{cl}^{*} A$ such that $g$ is upper semicontinuous, $h$ is lower semicontinuous, $g \leq f \leq h$, and $\int_{A}(h-g) d \mu<\varepsilon$. There is a positive function $\delta$ on $B$ such that $g(y)-\varepsilon<f(x)<h(y)+\varepsilon$ for each $x, y \in \operatorname{cl}^{*} A$ with $|x-y|<\delta(x)$. Thus if $\left\{\left(A_{1}, x_{1}\right), \ldots,\left(A_{p}, x_{p}\right)\right\}$ is a $\delta$-fine partition in $A$, we have

$$
\int_{A_{i}} g d \mu-\varepsilon G\left(A_{i}\right) \leq f\left(x_{i}\right) G\left(A_{i}\right) \leq \int_{A_{i}} h d \mu+\varepsilon G\left(A_{i}\right)
$$

and

$$
\int_{A_{i}} g d \mu \leq F\left(A_{i}\right) \leq \int_{A_{i}} h d \mu
$$

for $i=1, \ldots, p$. Consequently

$$
\sum_{i=1}^{p}\left|f\left(x_{i}\right) G\left(A_{i}\right)-F\left(A_{i}\right)\right| \leq \int_{A}(h-g) d \mu+\varepsilon G(A)<\varepsilon[1+G(A)]
$$

and the proposition is proved. 
Remark 2.7 Let $G$ be a nonnegative charge in $\mathbb{R}^{m}$, and let $G^{*}$ be the Stieltjes measure in $\mathbb{R}^{m}$ induced by $G$ according to [18, Chapter III, Section 5]. It follows from [3, Lemma 4.4] that $V_{*} G(K)=G(K)=G^{*}(K)$ for each cell $K \subset \mathbb{R}^{m}$, and hence $V_{*} G(U)=G^{*}(U)$ for every open set $U \subset \mathbb{R}^{m}$. As both measures $V_{*} G$ and $G^{*}$ are Borel regular (see [3, Lemma 4.6] and [18, Chapter III, Theorem 6.5]) and locally finite, they coincide [6, Section 1.1, Theorem 4(i)]. On the other hand, there is no simple relationship between the integral with respect to $G$ and the Lebesgue integral with respect to $G^{*}$. For instance, in the context of Example 2.2, we have $\int_{C} \chi_{C} d G=0$ while $\int_{C} \chi_{C} d G^{*}=1$.

Proposition 2.8 Let $G$ be a charge in $A \in B V_{c}$, and let $f$ be a function on $\mathrm{cl}^{*} A$. If $A$ is the union of a pair of disjoint $B V$ sets $B, C$ and $f \in \mathcal{R}(B ; G) \cap$ $\mathcal{R}(C ; G)$, then $f \in \mathcal{R}(A ; G)$ and $\int_{A} f d G=\int_{B} f d G+\int_{C} f d G$.

Proof. Let $F_{B}$ and $F_{C}$ be, respectively, the indefinite integrals of $f$ in $B$ and $C$ with respect to $G$. Then $F=F_{B} \mathrm{~L} B+F_{C} \mathrm{~L} C$ is a charge in $\mathbb{R}^{m}$, and it suffices to show that $F \uparrow B V(A)$ is the indefinite integral of $f$ in $A$ with respect to $G$.

To this end, let $\mathcal{B}$ and $\mathcal{C}$ be, respectively, the kernels of $B$ and $C$ associated with $f$ according to Definition 2.1, and choose an $X \in \mathcal{B}, Y \in \mathcal{C}$, and $\varepsilon>0$. There are gauges $\delta_{X}$ on $\mathrm{cl}^{*} X$ and $\delta_{Y}$ on $\mathrm{cl}^{*} Y$ such that

$$
\sum_{i=1}^{r}\left|f\left(y_{i}\right) G\left(B_{i}\right)-F_{B}\left(B_{i}\right)\right|<\varepsilon \quad \text { and } \sum_{i=1}^{s}\left|f\left(z_{i}\right) G\left(C_{i}\right)-F_{C}\left(C_{i}\right)\right|<\varepsilon
$$

for each $\varepsilon$-regular $\delta_{B}$-fine partition $\left\{\left(B_{1}, y_{1}\right), \ldots,\left(B_{r}, y_{r}\right)\right\}$ in $X$ and each $\varepsilon$ regular $\delta_{C}$-fine partition $\left\{\left(C_{1}, z_{1}\right), \ldots,\left(C_{s}, z_{s}\right)\right\}$ in $Y$. Making $\delta_{X}$ and $\delta_{Y}$ smaller, we can achieve that $B\left[x, \delta_{X}(x)\right]$ is disjoint from $Y$ for every $x \in$ $\mathrm{cl}^{*} X-\operatorname{cl} Y$, and $B\left[y, \delta_{Y}(y)\right]$ is disjoint from $X$ for every $y \in \operatorname{cl}^{*} Y-\operatorname{cl} X$. In view of Lemma 1.4, we may assume $X$ and $Y$ are nondispersed. As $X \cap Y=\emptyset$, we have

$$
(\operatorname{cl} X) \cap(\operatorname{cl} Y)=\left(\operatorname{cl}^{*} X\right) \cap\left(\operatorname{cl}^{*} Y\right) \subset\left(\partial^{*} X\right) \cap\left(\partial^{*} Y\right)
$$

and the set $(\operatorname{cl} X) \cap(\operatorname{cl} Y)$ is thin. Thus letting

$$
\delta(x)= \begin{cases}\delta_{X}(x) & \text { if } x \in \mathrm{cl}^{*} X-\operatorname{cl} Y \\ \delta_{Y}(x) & \text { if } x \in \mathrm{cl}^{*} Y-\operatorname{cl} X \\ 0 & \text { if } x \in(\operatorname{cl} X) \cap(\operatorname{cl} Y)\end{cases}
$$


defines a gage $\delta$ on $\operatorname{cl}^{*}(X \cup Y)$. Now if $P=\left\{\left(A_{1}, x_{1}\right), \ldots,\left(A_{p}, x_{p}\right)\right\}$ is an $\varepsilon$-regular $\delta$-fine partition in $X \cup Y$, then $P_{X}=\left\{\left(A_{i}, x_{i}\right): x_{i} \in \mathrm{cl}^{*} X\right\}$ is an $\varepsilon$ regular $\delta_{X}$-fine partition in $X$ and $P_{Y}=\left\{\left(A_{i}, x_{i}\right): x_{i} \in \mathrm{cl}^{*} Y\right\}$ is an $\varepsilon$-regular $\delta_{Y}$-fine partition in $Y$. As $P$ is the disjoint union of $P_{X}$ and $P_{Y}$, we obtain

$$
\begin{aligned}
\sum_{i=1}^{p}\left|f\left(x_{i}\right) G\left(A_{i}\right)-F\left(A_{i}\right)\right|= & \sum_{x_{i} \in \mathrm{Cl}^{*} X}\left|f\left(x_{i}\right) G\left(A_{i}\right)-F_{B}\left(A_{i}\right)\right| \\
& +\sum_{x_{i} \in \mathrm{Cl}^{*} Y}\left|f\left(x_{i}\right) G\left(A_{i}\right)-F_{C}\left(A_{i}\right)\right|<2 \varepsilon .
\end{aligned}
$$

Since $\mathcal{B} \vee \mathcal{C}$ is a kernel of $A$, the proposition follows.

Proposition 2.9 Let $G$ be a charge in $A \in B V_{c}$, let $h \in \mathcal{R}(A ; G)$, and let $H$ be the indefinite integral of $h$ in $A$ with respect to $G$. A function $f$ on $A$ belongs to $\mathcal{R}(A ; H)$ or $\mathcal{R}_{s}(A ; H)$ if and only if fh belongs to $\mathcal{R}(A ; G)$ or $\mathcal{R}_{s}(A ; G)$, respectively, in which case $\int_{A} f d H=\int_{A} f h d G$.

Proof. Let $f \in \mathcal{R}(A ; H)$ and let $F$ be the indefinite integral of $f$ in $A$ with respect to $H$. If $\mathcal{A}_{h}$ and $\mathcal{A}_{f}$ are kernels of $A$ associated with $h$ and $f$, respectively, according to Definition 2.1, then $\mathcal{A}=\mathcal{A}_{h} \wedge \mathcal{A}_{f}$ is a kernel of $A$ associated simultaneously with both $h$ and $f$. Choose a $B \in \mathcal{A}$ and an $\varepsilon>0$, and for $n=1,2, \ldots$, find a gage $\delta_{n}$ on $\mathrm{cl}^{*} B$ so that

$$
\sum_{i=1}^{p}\left|h\left(x_{i}\right) G\left(B_{i}\right)-H\left(B_{i}\right)\right|<\frac{\varepsilon}{n 2^{n}}
$$

for each $\varepsilon$-regular $\delta_{n}$-fine partition $\left\{\left(B_{1}, x_{1}\right), \ldots,\left(B_{p}, x_{p}\right)\right\}$ in $B$. If

$$
E_{n}=\left\{x \in \operatorname{cl}^{*} B: n-1 \leq|f(x)|<n\right\},
$$

then $\mathrm{cl}^{*} B$ is the disjoint union of the sets $E_{1}, E_{2}, \ldots$ Define a gage $\delta$ on $\mathrm{cl}^{*} B$ by letting $\delta(x)=\delta_{n}(x)$ for $x \in E_{n}$. Making $\delta$ smaller, we may assume

$$
\sum_{i=1}^{p}\left|f\left(x_{i}\right) H\left(B_{i}\right)-F\left(B_{i}\right)\right|<\varepsilon
$$

for each $\varepsilon$-regular $\delta$-fine partition $P=\left\{\left(B_{1}, x_{1}\right), \ldots,\left(B_{p}, x_{p}\right)\right\}$ in $B$. If $P$ is 
such a partition, we obtain

$$
\begin{aligned}
\sum_{i=1}^{p} \mid f\left(x_{i}\right) & h\left(x_{i}\right) G\left(B_{i}\right)-F\left(B_{i}\right) \mid \\
\leq & \sum_{i=1}^{p}\left|f\left(x_{i}\right) h\left(x_{i}\right) G\left(B_{i}\right)-f\left(x_{i}\right) H\left(B_{i}\right)\right|+\sum_{i=1}^{p}\left|f\left(x_{i}\right) H\left(B_{i}\right)-F\left(B_{i}\right)\right| \\
& <\sum_{n=1}^{\infty} \sum_{x_{i} \in E_{n}}\left|f\left(x_{i}\right)\right| \cdot\left|h\left(x_{i}\right) G\left(B_{i}\right)-H\left(B_{i}\right)\right|+\varepsilon<\sum_{n=1}^{\infty} n \frac{\varepsilon}{n 2^{n}}+\varepsilon \\
& =2 \varepsilon
\end{aligned}
$$

Thus $f h \in \mathcal{R}(A ; G)$ and $F$ is the indefinite integral of $f h$ in $A$ with respect to $G$. The proof of the converse is similar, and the modification of the previous argument for the case of $s$-integrability is obvious.

Let $\Omega \subset \mathbb{R}^{m}$ be an open set, and let $F$ be a function defined on $B V_{c}(\Omega)$. We say $F$ is derivable at $x \in \Omega$ if there is a finite $\operatorname{limit} \lim \frac{F\left(B_{n}\right)}{\left|B_{n}\right|}$ for each sequence $\left\{B_{n}\right\}$ in $B V_{c}(\Omega)$ such that $x \in \mathrm{cl}^{*} B_{n}, n=1,2, \ldots, \lim d\left(B_{n}\right)=0$, and $\inf r\left(B_{n}\right)>0$. When all such limits exist, they have the same value, denoted by $F^{\prime}(x)$ and called the derivate of $F$ at $x$.

Remark 2.10 Let $F$ be a charge in $A \in B V_{c}$, and let $x \in \operatorname{int}^{c} A$. According to [3, Lemma 3.5], the canonical extension $F \mathrm{~L} A$ is derivable at $x$ if and only if there is a finite limit $\lim \frac{F\left(B_{n}\right)}{\left|B_{n}\right|}$ for each sequence $\left\{B_{n}\right\}$ in $B V(A)$ such that $x \in \mathrm{cl}^{*} B_{n}, n=1,2, \ldots, \lim d\left(B_{n}\right)=0$, and $\inf r\left(B_{n}\right)>0$. When all such limits exist, they have the same value equal to $(F \mathrm{~L} A)^{\prime}(x)$.

The relationship between the integral and derivate is described by the following theorem proved in [3, Theorem 5.3 and Lemma 4.10].

Theorem 2.11 If $F$ is a charge in $A \in B V_{c}$, then the following conditions are equivalent.

1. The measure $V_{*}(F \mathrm{~L} A)$ restricted to the subsets of $\mathrm{cl}^{*} A$ is absolutely continuous and $\sigma$-finite.

2. The derivate $(F \mathrm{~L} A)^{\prime}(x)$ is defined at almost all $x \in \mathrm{cl}^{*} A$, the function $f: x \mapsto(F \mathrm{~L} A)^{\prime}(x)$ extended arbitrarily to $\mathrm{cl}^{*} A$ belongs to $\mathcal{R}_{s}(A ; \lambda)$, and $F$ is the indefinite integral of $f$ in $A$ with respect to $\lambda$.

Question 2.12 Let $F$ be a charge in $\mathbb{R}^{m}$. Does the absolute continuity of $V_{*} F$ imply its $\sigma$-finiteness? 
Question 2.12 arises naturally from condition 1 of Theorem 2.11. If $m=1$, an affirmative answer follows from a recent result of Bongiorno, Di Piazza, and Skvortsov [2] (see [15] for more details). For $m \geq 2$, the question remains an open problem, which appears quite difficult.

Definition 2.13 A charge $G$ in $A \in B V_{c}$ is called primitive if there is a kernel $\mathcal{A}$ of $A$ satisfying the following condition: for each $B \in \mathcal{A}$, the measure $V_{*}(G \mathrm{~L} B)$ restricted to the subsets of $\mathrm{cl}^{*} B$ is absolutely continuous and $\sigma$ finite. If this condition is satisfied by the trivial kernel $\mathcal{A}=\{A\}$, we say $G$ is a simple charge.

Theorem 2.11 justifies the names introduced in the previous definition: a charge $G$ in $A \in B V_{c}$ is, respectively, primitive or simple if and only if it is the indefinite integral of an integrable or simply integrable function in $A$ with respect to $\lambda$. Each simple charge is primitive, however, the converse is false in dimensions greater than one [11, Example 5.21].

Proposition 2.14 In dimension one, there is no difference between regular, primitive, and simple charges.

Proof. Each simple charge is primitive, and each primitive charge is regular. We show that for $m=1$, a regular charge $G$ in $A \in B V_{c}$ is simple. To this end, select a negligible set $E \subset \mathrm{cl}^{*} A$ and, using Lemma 1.4, find a countable kernel $\left\{B_{n}\right\}$ of $A$ so that $V_{*}\left(G \mathrm{~L} B_{n}\right)\left(E \cap \mathrm{cl}^{*} B_{n}\right)=0$ for $n=1,2, \ldots$ With no loss of generality, we may assume that the sets $A$ and $B_{n}$ are figures. Given positive numbers $\varepsilon$ and $\eta<1 /(2 m)$, there are gauges $\delta_{n}$ on $E \cap B_{n}$ such that

$$
\sum_{i=1}^{s}\left|G\left(B_{n} \cap C_{i}\right)\right|<\varepsilon 2^{-n}
$$

for each $\eta$-regular $\delta_{n}$-fine partition $\left\{\left(C_{1}, z_{1}\right), \ldots,\left(C_{s}, z_{s}\right)\right\}$ anchored in $E \cap B_{n}$. Making $\delta_{n}$ smaller, we may achieve that $\delta_{n}=0$ on $\partial B_{n}$, and $B\left[x, \delta_{n}(x)\right] \subset B_{n}$ whenever $x \in \operatorname{int} B_{n}$. In view of [11, Proposition 8.7], we can define a gage on $E \cap A$ by letting

$$
\delta(x)= \begin{cases}\delta_{n}(x) & \text { if } x \in B_{n}-\bigcup_{k=1}^{n} B_{k}, \\ 0 & \text { if } x \in A-\bigcup_{n=1}^{\infty} B_{n} .\end{cases}
$$

Now if $P=\left\{\left(C_{1}, z_{1}\right), \ldots,\left(C_{s}, z_{s}\right)\right\}$ is an $\eta$-regular $\delta$-fine partition anchored in $E \cap A$, then

$$
P_{n}=\left\{\left(C_{i}, z_{i}\right) \in P: z_{i} \in B_{n}-\bigcup_{k=1}^{n} B_{k}\right\}
$$


is an $\eta$-regular $\delta_{n}$-fine partition in $B_{n}$ anchored in $E \cap B_{n}$, and $P=\bigcup_{n=1}^{\infty} P_{n}$. Thus

$$
\sum_{i=1}^{s}\left|G\left(A \cap C_{i}\right)\right|=\sum_{n=1}^{\infty} \sum_{\left(C_{i}, z_{i}\right) \in P_{n}}\left|G\left(B_{n} \cap C_{i}\right)\right|<\sum_{n=1}^{\infty} \varepsilon 2^{-n}=\varepsilon,
$$

end we conclude that $V_{*}(G \mathrm{~L} A)(E \cap A)=0$. In view of the above mentioned result in [2], the charge $G$ is simple.

Let $A \in B V_{c}$ and let $\Phi: A \rightarrow \mathbb{R}^{m}$ be a lipeomorphism. Then $\Phi$ has a unique extension to a lipeomorphism from $\mathrm{cl}^{*} A$ onto $\mathrm{cl}^{*} \Phi(A)$ [11, Lemma 6.5], still denoted by $\Phi$, and we let $B^{\bullet}=\Phi(B)$ for each $B \in B V(A)$. By [11, Lemmas 6.5 and 6.6], the map $B \mapsto B^{\bullet}$, also denoted by $\Phi$, is a bijection from $B V(A)$ onto $B V\left(A^{\bullet}\right)$. According to Kirszbraun's theorem [7, Theorems 2.10.43], the map $\Phi$ can be extended to a Lipschitz map $\Psi: \mathbb{R}^{m} \rightarrow \mathbb{R}^{m}$. If $\Psi$ is differentiable at $x \in A$, let $J_{\Phi}(x)=|\operatorname{det} D \Psi(x)|$ where $D \Psi(x)$ is the derivative of $\Psi$ at $x$. In view of Rademacher's theorem [6, Section 3.1.2, Theorem 2], $J_{\Phi}(x)$ is defined for almost all $x \in \mathrm{cl}^{*} A$, and by [14, Lemma 10.5.5], up to a negligible set, the function $J_{\Phi}: x \mapsto J_{\Phi}(x)$, called the Jacobian of $\Phi$, is determined uniquely by $\Phi$ and does not depend on the extension $\Psi$.

Proposition 2.15 Let $A \in B V_{c}$, let $\Phi: A \rightarrow \mathbb{R}^{m}$ be a lipeomorphism, and let $G$ be a charge in $\Phi(A)$. Then $G \circ \Phi$ is a charge in $A$, and if $f$ belongs to $\mathcal{R}(\Phi(A) ; G)$, then $f \circ \Phi$ belongs to $\mathcal{R}(A ; G \circ \Phi)$ and

$$
\int_{A} f \circ \Phi d(G \circ \Phi)=\int_{\Phi(A)} f d G
$$

Proof. If $x \in \mathrm{cl}^{*} A$ and $B \in B V(A)$, let $x^{\bullet}=\Phi(x)$ and $B^{\bullet}=\Phi(B)$. There are positive constants $a, b$ such that $a|x-y| \leq\left|x^{\bullet}-y^{\bullet}\right| \leq b|x-y|$ for all $x, y \in \mathrm{cl}^{*} A$. It follows that $a^{m}|B| \leq\left|B^{\bullet}\right| \leq b^{m}|B|$ and $a^{m-1}\|B\| \leq\left\|B^{\bullet}\right\| \leq b^{m-1}\|B\|$ for each $B \in B V(A)$, and we conclude that $G \circ \Phi$ is a charge in $A$.

Let $f \in \mathcal{R}\left(A^{\bullet} ; G\right)$, and let $F$ be the indefinite integral of $f$ in $A^{\bullet}$ with respect to $G$. If $\mathcal{A}^{\bullet}$ is a kernel of $A^{\bullet}$ associated with $f$ according to Definition 2.1, then $\mathcal{A}=\left\{B: B^{\bullet} \in \mathcal{A}^{\bullet}\right\}$ is a kernel of $A$. Choose a $B \in \mathcal{A}$ and an $\varepsilon>0$, and find a gage $\delta^{\bullet}$ on $\mathrm{cl}^{*} B^{\bullet}$ so that $\sum_{i=1}^{p}\left|f\left(x_{i}^{\bullet}\right) G\left(B_{i}^{\bullet}\right)-F\left(B_{i}^{\bullet}\right)\right|<\varepsilon$ for every $\varepsilon$-regular $\delta^{\bullet}$-fine partition $\left\{\left(B_{1}^{\bullet}, x_{1}^{\bullet}\right), \ldots,\left(B_{p}^{\bullet}, x_{p}^{\bullet}\right)\right\}$ in $B^{\bullet}$. Define a gage $\delta$ on $\mathrm{cl}^{*} B$ by letting $\delta(x)=\delta^{\bullet}\left(x^{\bullet}\right) / b$ for each $x \in \mathrm{cl}^{*} B$, and let $\varepsilon^{\prime}=(a / b)^{m} \varepsilon$. Now if $\left\{\left(B_{1}, x_{1}\right), \ldots,\left(B_{p}, x_{p}\right)\right\}$ is an $\varepsilon^{\prime}$-regular $\delta$-fine partition in $B$, then $\left\{\left(B_{1}^{\bullet}, x_{1}^{\bullet}\right)\right.$, $\left.\ldots,\left(B_{p}^{\bullet}, x_{p}^{\bullet}\right)\right\}$ is an $\varepsilon$-regular $\delta^{\bullet}$-fine partition in $B^{\bullet}$. Thus

$$
\sum_{i=1}^{p}\left|f \circ \Phi\left(x_{i}\right) G \circ \Phi\left(B_{i}\right)-F \circ \Phi\left(B_{i}\right)\right|=\sum_{i=1}^{p}\left|f\left(x_{i}^{\bullet}\right) G\left(B_{i}^{\bullet}\right)-F\left(B_{i}^{\bullet}\right)\right|<\varepsilon,
$$


and the proposition follows.

Corollary 2.16 Let $A \in B V_{c}$, and let $\Phi: A \rightarrow \mathbb{R}^{m}$ be a lipeomorphism. If $f$ belongs to $\mathcal{R}(\Phi(A) ; \lambda)$, then $(f \circ \Phi) J_{\Phi}$ belongs to $\mathcal{R}(A ; \lambda)$ and

$$
\int_{A}(f \circ \Phi) J_{\Phi} d \lambda=\int_{\Phi(A)} f d \lambda .
$$

Proof. By the area theorem [6, Section 3.1.2, Theorem 2] and Proposition 2.6, the Jacobian $J_{\Phi}$ of $\Phi$ belongs to $\mathcal{R}(A ; \lambda)$ and $\lambda \circ \Phi$ is the indefinite integral of $J_{\Phi}$ in $A$ with respect to $\lambda$. The corollary follows from Propositions 2.9 and 2.15 .

Let $C \subset \mathbb{R}^{m}$ and let $n \geq 1$ be an integer. We say a map $v: C \rightarrow \mathbb{R}^{n}$ is almost differentiable at $x \in C$ whenever there are positive numbers $c$ and $\delta$ such that $|v(y)-v(x)|<c|y-x|$ for each $y \in C$ with $|y-x|<\delta$.

Suppose $C$ is closed and $v$ is continuous, and denote by $E$ the set of all $x \in C$ at which $v$ is almost differentiable. A version of Whitney's extension theorem [20, Chapter 6, Section 2] shows that $v$ has a continuous extension $w$ : $\mathbb{R}^{m} \rightarrow \mathbb{R}^{n}$ such that $w$ is almost differentiable at each $x \in E$. By Stepanoff's theorem [7, Theorem 3.1.9], the map $w$ is differentiable almost everywhere in $E$. If $m=n$ we let $\operatorname{div} v(x)=\operatorname{div} w(x)$ for every $x \in E$ at which $w$ is differentiable. According to [14, Lemma 10.5.5], up to a negligible set, the divergence $\operatorname{div} v$ is determined uniquely by $v$ and does not depend on the extension $w$. The following divergence theorem has been established in [11, Theorems 5.19 and 9.13].

Theorem 2.17 Let $A \in B V_{c}$, and let $v: \operatorname{cl} A \rightarrow \mathbb{R}^{m}$ be a continuous vector field. Suppose there is a kernel $\mathcal{A}$ of $A$ with the following property: for each $B \in \mathcal{A}$ there is an thin set $T_{B}$ such that $v \uparrow \mathrm{cl} B$ is almost differentiable at every $x \in \mathrm{cl}^{*} B-T_{B}$. Then $\operatorname{div} v$ belongs to $\mathcal{R}(A ; \lambda)$ and

$$
\int_{A} \operatorname{div} v d \lambda=\int_{\partial^{*} A} v \cdot \nu_{A} d \mathcal{H} .
$$

Moreover, if the kernel $\mathcal{A}$ is trivial, then $\operatorname{div} v$ belongs to $\mathcal{R}_{s}(A ; \lambda)$.

\section{$3 \quad$ Integrable Functions}

Given a set $A \in B V_{c}$, we shall study the space $\mathcal{R}(A)=\bigcap \mathcal{R}(A ; G)$ where the intersection is taken over all charges $G$ in $A$. Our first result shows that $\mathcal{R}(A) \subset \mathcal{B V}^{\infty}\left(\mathrm{cl}^{*} A\right)$. 
Proposition 3.1 Let $A \in B V_{c}$, and let $f$ be a function on $\mathbb{R}^{m}$ that vanishes outside $\mathrm{cl}^{*} A$. Then $f \in \mathcal{B V}^{\infty}\left(\mathrm{cl}^{*} A\right)$ whenever either of the following conditions holds:

1. $f \in \mathcal{R}(A ; G)$ for each primitive charge $G$ in $A$;

2. $f \in \mathcal{R}_{s}(A ; G)$ for each simple charge $G$ in $A$.

Proof. It suffices to prove the proposition for $m \geq 2$; the case $m=1$ follows from [19]. By our assumptions, $f$ belongs to $\mathcal{R}(A ; \lambda)$ or $\mathcal{R}_{s}(A ; \lambda)$, and we denote by $F$ the indefinite integral of $f$ in $A$ with respect to $\lambda$. Again by our assumptions, $f \in \mathcal{R}(A ; F)$, and hence $f^{2} \in L^{1}(A ; \lambda)$ by Proposition 2.9 and [11, Proposition 9.5,5]. As $L^{2}(A ; \lambda) \subset L^{1}(A ; \lambda)$, the function $f$ belongs to $L^{1}\left(\mathbb{R}^{m} ; \lambda\right)$.

Next assume $f$ is not a $B V$ function, and find real numbers $a<b$ so that $A$ is contained in the cell $K=[a, b]^{m}$. If $a<t<b$ let

$$
K_{t-}=[a, t] \times[a, b]^{m-1} \text { and } K_{t+}=[t, b] \times[a, b]^{m-1} .
$$

There is a $v_{1} \in C_{c}^{1}\left(\mathbb{R}^{m}, \mathbb{R}^{m}\right)$ with $\left\|v_{1}\right\|_{\infty} \leq 1$ and $\int_{A} f \operatorname{div} v_{1} d \lambda>2^{3}$. Find a $t \in(a, b)$ so that $\int_{A \cap K_{t \pm}} f \operatorname{div} v_{1} d \lambda=\frac{1}{2} \int_{A} f \operatorname{div} v_{1} d \lambda>2^{2}$, and denote by $K_{1}$ that cell from the pair $\left\{K_{t-}, K_{t+}\right\}$ for which the function $f \chi_{A-K_{1}}$ is not $B V$. There is a $v_{2} \in C_{c}^{1}\left(\mathbb{R}^{m}, \mathbb{R}^{m}\right)$ with $\left\|v_{2}\right\|_{\infty} \leq$ and $\int_{A-K_{1}} f \operatorname{div} v_{2} d \lambda>2^{5}$. Repeating the previous argument with $K$ replaced by $\operatorname{cl}\left(K-K_{1}\right)$, find a cell $K_{2} \subset \operatorname{cl}\left(K-K_{1}\right)$ so that $\int_{A \cap K_{2}} f \operatorname{div} v_{2} d \lambda>2^{4}$ and $f \chi_{A-\left(K_{1} \cup K_{2}\right)}$ is not $B V$. Proceeding inductively, construct sequences $\left\{K_{n}\right\}$ of cells and $\left\{v_{n}\right\}$ of vector fields in $C_{c}^{1}\left(\mathbb{R}^{m}, \mathbb{R}^{m}\right)$ such that $\left\|v_{n}\right\|_{\infty} \leq 1$ and $\int_{A \cap K_{n}} f \operatorname{div} v_{n} d \lambda>2^{2 n}$. It is clear from the construction that $K_{n}=\left[t_{n}, t_{n+1}\right] \times[a, b]^{m-1}$ are nonoverlapping cells, and that the sequence $\left\{t_{n}\right\}$ has at most two cluster points $\alpha$ and $\beta$. For $n=1,2, \ldots$ and $i=1, \ldots, 2^{n}$, let

$$
L_{n, i}=\left[t_{n}, t_{n+1}\right] \times\left[a+\frac{i-1}{2^{n}}(b-a), a+\frac{i}{2^{n}}(b-a)\right] \times[a, b]^{m-2} .
$$

Since $L_{n, 1}, \ldots, L_{n, 2^{n}}$ are nonoverlapping cells whose union is $K_{n}$, there is an $i(n)$ with $1 \leq i(n) \leq 2^{n}$ and $\int_{A \cap L_{n, i(n)}} f \operatorname{div} v_{n} d \lambda>2^{n}$. Now select cells $L_{n} \subset$ int $L_{n, i(n)}$ so that $\int_{A \cap L_{n}} f \operatorname{div} v_{n} d \mathcal{H}^{m}>2^{n}$, and observe that with no loss of generality we may assume $\operatorname{supp} v_{n} \subset L_{n, i(n)}$. The vector field $v=\sum_{n=1}^{\infty} 2^{-n} v_{n}$ is continuous in $\mathbb{R}^{m}$ and differentiable outside the thin set $\left\{\left(\xi_{1}, \ldots, \xi_{m}\right) \in \mathbb{R}^{m}\right.$ : $\xi_{1}=\alpha$ or $\left.\xi_{1}=\beta\right\}$. According to the divergence theorem (Theorem 2.17), we can define a simple charge $G$ in $\mathbb{R}^{m}$ by letting $G(C)=\int_{C} \operatorname{div} v d \lambda$ for every 
$C \in B V_{c}$. Set $B_{k}=A \cap \bigcup_{n=1}^{k} L_{n}$, and observe that the sequence $\left\{B_{k}\right\} \tau$ converges to $B=A \cap \bigcup_{n=1}^{\infty} L_{n}$; indeed,

$$
\left\|B_{k}\right\| \leq\|A\|+\sum_{n=1}^{k}\left\|L_{n}\right\|<\|A\|+\sum_{n=1}^{\infty}\left\|L_{n, i(n)}\right\|
$$

and an easy calculation reveals that $\sum_{n=1}^{\infty}\left\|L_{n, i(n)}\right\|<\|K\|$. Since $f$ belongs to $\mathcal{R}(A ; G)$ by our assumptions, Proposition 2.9 implies

$$
\begin{aligned}
\int_{B} f d G & =\int_{B} f \operatorname{div} v d \lambda=\lim _{k \rightarrow \infty} \sum_{n=1}^{k} \int_{A \cap L_{n}} f \operatorname{div} v d \lambda \\
& =\lim _{k \rightarrow \infty} \sum_{n=1}^{k} 2^{-n} \int_{A \cap L_{n}} f \operatorname{div} v_{n} d \lambda=+\infty,
\end{aligned}
$$

a contradiction.

Finally, if $f$ is a $B V$ function with $\|f\|_{\infty}=+\infty$, we may assume by symmetry that $\left\|f^{+}\right\|_{\infty}=+\infty$. According to the coarea formula for $B V$ functions [6, Section 5.5, Theorem 1], for $n=1,2, \ldots$, there are real numbers $s_{n} \geq 2^{n}$ such that $C_{n}=\left\{x \in A: f(x)>s_{n}\right\}$ are $B V$ sets of positive measure with $\left\|C_{n}\right\| \leq 1$. Letting $\mu(E)=\sum_{n=1}^{\infty} 2^{-n} \frac{\left|E \cap C_{n}\right|}{\left|C_{n}\right|}$ for each $E \subset \mathbb{R}^{m}$ defines a finite absolutely continuous measure $\mu$ in $\mathbb{R}^{m}$. Thus $H=\mu \uparrow B V(A)$ is a simple charge in $A$, and $f \in \mathcal{R}(A ; H)$ by our assumptions. As $\lim \left|C_{k}\right|=0$ and $\int_{C_{k}} f d H \geq s_{k} H\left(C_{k}\right) \geq 1$ for $k=1,2, \ldots$, we again have a contradiction.

If $A \in B V_{c}$ and $C \in B V(A \times \mathbb{R})$, we let $C^{t}=\{x \in A:(x, t) \in C\}$. It follows from [8, Theorems 20 and 33] that $C^{t} \in B V(A)$ for almost all $t \in \mathbb{R}$.

Lemma 3.2 Let $G$ be a charge in $A \in B V_{c}$. If $C \in B V(A \times \mathbb{R})$, then the function $t \mapsto G\left(C^{t}\right)$, defined for almost all $t \in \mathbb{R}$, belongs to $L^{1}(\mathbb{R} ; \lambda)$. Letting $\hat{G}(C)=\int_{\mathbb{R}} G\left(C^{t}\right) d \lambda(t)$ for each $C \in B V(A \times \mathbb{R})$ defines a charge $\hat{G}$ in $A \times \mathbb{R}$.

Proof. Select a $C \in B V(A \times \mathbb{R}), \varepsilon>0$, and real numbers $a<b$ so that $C \subset A \times[a, b]$. By Lemma 1.2, there is a $\theta>0$ such that

$$
\left|G\left(C^{t}\right)\right|<\theta\left|C^{t}\right|+\varepsilon\left[\left\|C^{t}\right\|+\chi_{[a, b]}(t)\right]
$$

for almost all $t \in \mathbb{R}$.

Let $L$ be the linear space of all functions $f$ on $\mathbb{R}$ for which there is a $g \in L^{1}(\mathbb{R} ; \lambda)$ with $|f| \leq g$. By the Hahn-Banach theorem [17, Theorem 3.2], there is a linear functional $\Lambda: L \rightarrow \mathbb{R}$ such that $\Lambda(f) \leq \int_{\mathbb{R}} g d \lambda$ for each 
$f \in L$ and each $g \in L^{1}(\mathbb{R} ; \lambda)$ with $f \leq g$; in particular, $\Lambda$ is nonnegative and $\Lambda(g)=\int_{A} g d \lambda$ whenever $g \in L^{1}(\mathbb{R} ; \lambda)$. For $t \in \mathbb{R}$, let

$$
f_{C}(t)= \begin{cases}G\left(C^{t}\right) & \text { if } C^{t} \in B V(A), \\ 0 & \text { otherwise. }\end{cases}
$$

By Fubini's theorem and [9, Section 2.2.1],

$$
\int_{\mathbb{R}}\left|C^{t}\right| d \lambda(t)=|C| \text { and } \int_{\mathbb{R}}\left\|C^{t}\right\| d \lambda(t) \leq\|C\|,
$$

from which we conclude $H(C)=\Lambda\left(f_{C}\right)$ is a well defined real number. Thus we have obtained an additive function $H$ in $A \times \mathbb{R}$. Applying $\Lambda$ to inequality (3.1) yields

$$
|H(C)|<\theta|C|+\varepsilon(\|C\|+b-a)
$$

so $H$ is a charge. We claim that $H(C)$ does not depend on the choice of $\Lambda$.

If $K$ is a figure and $\hat{K}=K \cap(A \times[a, b])$, then

$$
H(\hat{K})=\int_{\mathbb{R}} G\left(\hat{K}^{t}\right) d \lambda(t),
$$

as $t \mapsto G\left(\hat{K}^{t}\right)$ is a simple measurable function with compact support. By DeGiorgi's approximation theorem [3, Proposition 1.1], there is a sequence $\left\{K_{i}\right\}$ of figures such that $\lim \left|\left(K_{i}-C\right) \cup\left(C-K_{i}\right)\right|=0$ and $\sup \left\|K_{i}\right\|<+\infty$. Clearly, the same conditions are satisfied by the sequence $\left\{\widehat{K}_{i}\right\}$. From

$$
\left|H\left(\widehat{K_{i}}\right)-H(C)\right|=\left|H\left(\widehat{K_{i}}-C\right)-H\left(C-\widehat{K_{i}}\right)\right| \leq\left|H\left(\widehat{K_{i}}-C\right)\right|+\left|H\left(C-\widehat{K}_{i}\right)\right|
$$

it follows that $H(C)=\lim H\left(\widehat{K_{i}}\right)$, and the claim is proved.

Using the claim, it is easy to deduce from the construction of $\Lambda$ that the function $f_{C}$ is measurable. Thus $H=\hat{G}$, which establishes the lemma.

Remark 3.3 The measurability of the function $f_{C}$ defined in the proof of Lemma 3.2 can be established directly (i.e., without referring to the HahnBanach theorem) by the following argument of J. Král.

Observe that $g_{i}: t \mapsto G\left(\widehat{K}_{i}^{t}\right)$ is a simple measurable function on $[a, b]$; for $\widehat{K}_{i}^{t}=K_{i}^{t} \cap A$ for each $t \in[a, b]$. Since $\lim _{i, j \rightarrow \infty}\left|\left(\widehat{K}_{i}-\widehat{K}_{j}\right) \cup\left(\widehat{K}_{j}-\widehat{K}_{i}\right)\right|=0$ and $\sup \left\|\widehat{K}_{i}\right\|<+\infty$, from inequality (3.1) follows $\lim _{i, j \rightarrow \infty} \int_{a}^{b}\left|g_{i}-g_{j}\right| d \lambda=0$. Thus $\left\{g_{i}\right\}$ converges in $L^{1}([a, b] ; \lambda)$ to a function $g \in L^{1}([a, b] ; \lambda)$, and it 
suffices to show that $f_{C}(t)=g(t)$ for almost all $t \in[a, b]$. According to Fubini's theorem, the functions $h_{i}: t \mapsto\left|\left(\widehat{K}_{i}^{t}-C^{t}\right) \cup\left(C^{t}-\widehat{K}_{i}^{t}\right)\right|$ converge to zero in $L^{1}([a, b] ; \lambda)$. Passing to a subsequence, there is a negligible set $N \subset[a, b]$ such that $\lim g_{i}(t)=g(t)$ and $\lim h_{i}(t)=0$ for each $t \in[a, b]-N$. By Fatou's lemma,

$$
\int_{a}^{b} \liminf \left\|\widehat{K}_{i}^{t}\right\| d \lambda(t) \leq \liminf \left\|\widehat{K}_{i}\right\| \leq \sup \left\|\widehat{K}_{i}\right\|<+\infty .
$$

Enlarging $N$, we can achieve that $\liminf \left\|\widehat{K}_{i}^{t}\right\|<+\infty$ for every $t \in[a, b]-$ $N$. Now fix a $t \in[a, b]-N$, and find a subsequence $\left\{K_{i_{j}}\right\}$ of $\left\{K_{i}\right\}$ with $\sup \left\|{\widehat{K_{i_{j}}}}^{t}\right\|<+\infty$. It follows that $\left\{{\widehat{K_{i_{j}}}}^{t}\right\} \tau$-converges to $C^{t}$. We conclude

$$
f_{C}(t)=G\left(C^{t}\right)=\lim G\left({\widehat{K_{i_{j}}}}^{t}\right)=\lim g_{i_{j}}(t)=g(t),
$$

which completes the argument.

Let $f$ be a nonnegative function on $A$, and let $E \subset A$. The subgraph of $f$ over $E$ is the set

$$
\Sigma(f, E)=\{(x, t) \in E \times \mathbb{R}: 0 \leq t \leq f(x)\} .
$$

The following theorem is a mild generalization of the result obtained in [10].

Theorem 3.4 Let $G$ be a charge in $A \in B V_{c}$, and let $f$ be a bounded $B V$ function. If there is a thin set $T$ such that $f\left\lceil\mathrm{cl}^{*} A\right.$ is almost differentiable at each $x \in \mathrm{cl}^{*} A-T$, then $f \in \mathcal{R}_{s}(A ; G)$ and

$$
\int_{A} f d G=\hat{G}\left[\Sigma\left(f^{+}, A\right)\right]-\hat{G}\left[\Sigma\left(f^{-}, A\right)\right] .
$$

Proof. For $x \in \mathrm{cl}^{*} A-T$ let

$$
h(x)=\limsup _{\substack{y \rightarrow x \\ y \in \mathrm{cl}^{*} A}} \frac{|f(y)-f(x)|}{|y-x|},
$$

and set $h(x)=0$ for each $x \in T \cap \mathrm{cl}^{*} A$. This defines a nonnegative measurable function $h$ on $\mathrm{cl}^{*} A$, which is finite by our assumption. Analyzing the proof of Stepanoff's theorem [14, Theorem 6.6.8], it is easy to deduce that almost everywhere in $\mathrm{cl}^{*} A-T$, the function $f$ is differentiable "relative" to $\mathrm{cl}^{*} A$. More precisely, there is a negligible set $N$ containing $T$, and a vector field 
$\nabla f: \mathrm{cl}^{*} A-N \rightarrow \mathbb{R}^{m}$ with the following property: given $x \in \mathrm{cl}^{*} A-N$ and $\varepsilon>0$, we can find a $\delta>0$ so that

$$
|f(y)-f(x)-\nabla f(x) \cdot(y-x)|<\varepsilon|y-x|
$$

for each $y \in B[x, \delta] \cap \mathrm{cl}^{*} A$. It follows $f$ is approximately differentiable at each $x \in$ int $^{*} A-N$. According to [6, Section 6.1.3, Theorem 4 and the following Remark], almost everywhere in $\mathrm{cl}^{*} A$, the vector field $\nabla f$ equals the density of the absolutely continuous part of $D f$. The previous inequality yields

$$
\frac{|f(y)-f(x)|}{|y-x|}<\varepsilon+\sqrt{m}\|\nabla f(x)\|
$$

for all $y \in B[x, \delta] \cap \mathrm{cl}^{*} A$. As $\varepsilon$ is arbitrary, $h(x) \leq \sqrt{m}\|\nabla f(x)\|$ for each $x \in \operatorname{cl}^{*} A-N$; in particular,

$$
\int_{A} h d \lambda \leq \sqrt{m} \int_{A}\|\nabla f\| d \lambda \leq \sqrt{m}\|f\| .
$$

The rest of the proof parallels that of the main theorem of [10]. With no loss of generality, suppose $0 \leq f \leq 1$, and for each $B \in B V(A)$, let $\Sigma_{B}=\Sigma(f, B)$. Observe that $\left|\Sigma_{B}\right| \leq|B|$ and

$$
\left\|\Sigma_{B}\right\| \leq\|B\|+2|B|+\|D f\|(B) \leq\|B\|+2|A|+\|f\| .
$$

Thus $\Sigma_{B} \in B V(A \times[0,1])$ for each $B \in B V(A)$ and, in view of Lemma 3.2, the map $F: B \mapsto \hat{G}\left(\Sigma_{B}\right)$ is a charge in $A$. Choose an $\varepsilon>0$ and find an $\eta>0$ so that $|\hat{G}(C)|<\varepsilon$ for every $B V$ set $C \subset A \times[0,1]$ with

$$
|C|<\eta(1+\sqrt{m}\|f\|+|A|)
$$

and

$$
\|C\|<2|A|+\|f\|+\frac{1}{\varepsilon}(1+\sqrt{m}\|f\|+|A|) .
$$

For each $x \in \operatorname{cl}^{*} A-T$, there is a positive $\delta_{x}<\eta$ such that

$$
|f(y)-f(x)|<(h(x)+1)|y-x|
$$

for all $y \in B\left[x, \delta_{x}\right] \cap \mathrm{cl}^{*} A$. Extending the map $x \mapsto \delta_{x}$ to $\mathrm{cl}^{*} A$ by zero defines a gage $\delta$ on $\mathrm{cl}^{*} A$. In view of inequality (3.2) and Proposition 2.6, making $\delta$ smaller, we may assume

$$
\sum_{i=1}^{p} h\left(x_{i}\right)\left|A_{i}\right|<\sqrt{m}\|f\|+1
$$


for each $\varepsilon$-regular $\delta$-fine partition $\left\{\left(A_{1}, x_{1}\right), \ldots,\left(A_{p}, x_{p}\right)\right\}$ in $A$. Choose such a partition, and let $J_{i}=\left[0, f\left(x_{i}\right)\right]$ for $i=1, \ldots, p$. After a suitable reordering, we find a nonnegative integer $k \leq p$ so that

$$
\begin{aligned}
S= & \sum_{i=1}^{p}\left|f\left(x_{i}\right) G\left(A_{i}\right)-F\left(A_{i}\right)\right| \\
= & \left|\sum_{i=1}^{k}\left[\hat{G}\left(A_{i} \times J_{i}\right)-\hat{G}\left(\Sigma_{A_{i}}\right)\right]\right|+\left|\sum_{i=k+1}^{p}\left[\hat{G}\left(A_{i} \times J_{i}\right)-\hat{G}\left(\Sigma_{A_{i}}\right)\right]\right| \\
\leq & \left|\hat{G}\left[\bigcup_{i=1}^{k}\left(A_{i} \times J_{i}-\Sigma_{A_{i}}\right)\right]\right|+\left|\hat{G}\left[\bigcup_{i=1}^{k}\left(\Sigma_{A_{i}}-A_{i} \times J_{i}\right)\right]\right| \\
& +\left|\hat{G}\left[\bigcup_{i=k+1}^{p}\left(A_{i} \times J_{i}-\Sigma_{A_{i}}\right)\right]\right|+\left|\hat{G}\left[\bigcup_{i=k+1}^{p}\left(\Sigma_{A_{i}}-A_{i} \times J_{i}\right)\right]\right| .
\end{aligned}
$$

From inequality (3.3), we infer

$$
\begin{aligned}
& \left|\bigcup_{i=r}^{s}\left(A_{i} \times J_{i}-\Sigma_{A_{i}}\right)\right|=\sum_{i=r}^{s}\left|A_{i} \times J_{i}-\Sigma_{A_{i}}\right| \\
& \quad=\sum_{i=r}^{s} \int_{A_{i}}\left|f(x)-f\left(x_{i}\right)\right| d \lambda(x) \leq \sum_{i=r}^{s}\left(h\left(x_{i}\right)+1\right) d\left(A_{i}\right)\left|A_{i}\right| \\
& \quad \leq \eta \sum_{i=r}^{s}\left(h\left(x_{i}\right)+1\right)\left|A_{i}\right|<\eta(1+\sqrt{m}\|f\|+|A|)
\end{aligned}
$$

and

$$
\begin{aligned}
& \left\|\bigcup_{i=r}^{s}\left(A_{i} \times J_{i}-\Sigma_{A_{i}}\right)\right\| \leq \sum_{i=r}^{s}\left\|A_{i} \times J_{i}-\Sigma_{A_{i}}\right\| \\
& \quad \leq \sum_{i=r}^{s}\left[2\left|A_{i}\right|+\|D f\|\left(A_{i}\right)+\int_{\partial^{*} A_{i}}\left|f(x)-f\left(x_{i}\right)\right| d \mathcal{H}(x)\right] \\
& \quad \leq 2|A|+\|f\|+\sum_{i=r}^{s}\left(h\left(x_{i}\right)+1\right) d\left(A_{i}\right)\left\|A_{i}\right\| \\
& \quad<2|A|+\|f\|+\frac{1}{\varepsilon} \sum_{i=r}^{s}\left(h\left(x_{i}\right)+1\right)\left|A_{i}\right| \\
& \quad<2|A|+\|f\|+\frac{1}{\varepsilon}(1+\sqrt{m}\|f\|+|A|)
\end{aligned}
$$


for all integers $r, s$ with $1 \leq r \leq s \leq p$. As the same estimates are true for the sets $\bigcup_{i=r}^{s}\left(\Sigma_{A_{i}}-A_{i} \times J_{i}\right)$, we conclude $S<4 \varepsilon$ and the theorem is proved.

Corollary 3.5 Let $G$ be a regular charge in $A \in B V_{c}$, and let $f$ be an essentially bounded $B V$ function. Suppose there exists a kernel $\mathcal{A}$ of $A$ with the following property: for each $B \in \mathcal{A}$ there is a thin set $T_{B}$ such that $f\left\lceil\mathrm{cl}^{*} B\right.$, modified on a negligible set, is almost differentiable at each $x \in \mathrm{cl}^{*} B-T_{B}$. Then $f \in \mathcal{R}(A ; G)$ and

$$
\int_{A} f d G=\hat{G}\left[\Sigma\left(f^{+}, A\right)\right]-\hat{G}\left[\Sigma\left(f^{-}, A\right)\right] .
$$

In particular, the conclusion holds when $f$ is a simple $B V$ function.

Proof. Since $G$ is a regular charge, Theorem 3.4 implies $f \in \mathcal{R}(B ; G)$ and

$$
\int_{B} f d G=\hat{G}\left[\Sigma\left(f^{+}, B\right)\right]-\hat{G}\left[\Sigma\left(f^{-}, B\right)\right]
$$

for each $B \in \mathcal{A}$. Observing that both sides of the previous equality are charges in $A$, and that the families $\left\{\Sigma\left(f^{+}, B\right): B \in \mathcal{A}\right\}$ and $\left\{\Sigma\left(f^{-}, B\right): B \in \mathcal{A}\right\}$ are kernels of $\Sigma\left(f^{+}, A\right)$ and $\Sigma\left(f^{-}, A\right)$, respectively, the first part of the corollary is established.

Let $f$ be a simple $B V$ function. Then $f\left\lceil\mathrm{cl}^{*} A=\sum_{k=1}^{n} c_{k} \chi_{A_{k}}\right.$ where $c_{1}, \ldots, c_{k}$ are distinct real numbers and $A_{1}, \ldots, A_{n}$ are disjoint $B V$ sets whose union equals $\mathrm{cl}^{*} A$. By Lemma 1.4, each $A_{k}$ has a nondispersed kernel $\mathcal{A}_{k}$, and the family $\mathcal{A}=\bigvee_{k=1}^{n} \mathcal{A}_{k}$ is the kernel of $A$. If $B_{k} \in \mathcal{A}_{k}$ for $k=1, \ldots, n$ and $B=\bigcup_{k=1}^{n} B_{k}$, let $g=\sum_{k=1}^{n} c_{k} \chi_{\mathrm{cl}^{*} B_{k}}$ and observe that $g=f$ almost everywhere in $\mathrm{cl}^{*} B$. Moreover, $g\left\lceil\mathrm{cl}^{*} B\right.$ is locally constant on $\mathrm{cl}^{*} B-\bigcup_{k=1}^{n} \partial^{*} B_{k}$; indeed, each $B_{i}-\partial^{*} B_{i}$ is disjoint from

$$
\bigcup_{\substack{k=1 \\ k \neq i}}^{n} \operatorname{cl}^{*} B_{k}=\bigcup_{\substack{k=1 \\ k \neq i}}^{n} \operatorname{cl} B_{k}=\operatorname{cl}\left(B-B_{i}\right) .
$$

As $\bigcup_{k=1}^{n} \partial^{*} B_{k}$ is a thin set, the corollary is proved.

Proposition 3.6 Let $G$ be a simple charge in $A \in B V_{c}$, and let $f$ be a simple $B V$ function. Then $f \in \mathcal{R}_{s}(A ; G)$ and

$$
\int_{A} f d G=\hat{G}\left[\Sigma\left(f^{+}, A\right)\right]-\hat{G}\left[\Sigma\left(f^{-}, A\right)\right] .
$$


Proof. Since both sides of the desired equality are linear functionals on the space of simple $B V$ functions, we may assume that $f$ is the characteristic function of a $B V$ set $B$. To simplify the notation, denote $G \mathrm{~L} A$ by $G$. Let $F=G \mathrm{~L} B$, and select a set $E \subset \mathrm{cl}^{*} A$ with $V_{*} G(E)<\alpha<+\infty$. Choose a positive $\eta<1 /(2 m)$, and set $\eta^{\prime}=\eta^{m+1}$. In view of Remark 2.3, there is a gage $\delta$ on $E$ such that $\sum_{i=1}^{p}\left|G\left(A_{i}\right)\right|<\alpha$ for each $\eta^{\prime}$-regular $\delta$-fine partition $\left\{\left(A_{1}, x_{1}\right), \ldots,\left(A_{p}, x_{p}\right)\right\}$ in $A$ anchored in $E$. We may assume that $\delta(x)=0$ for all $x \in E \cap \partial^{c} B$. Now on $E$ there is a gage $\delta^{\prime} \leq \delta$ such that if $\left\{\left(B_{1}, x_{1}\right)\right.$, $\left.\ldots,\left(B_{q}, x_{q}\right)\right\}$ is an $\eta$-regular $\delta^{\prime}$-fine partition in $A$ anchored in $E$, then

$$
\left\{\left(B_{i} \cap B, x_{i}\right): x_{i} \in \mathrm{cl}^{*} B\right\} \quad \text { and } \quad\left\{\left(B_{i}-B, x_{i}\right): x_{i} \notin \mathrm{cl}^{*} B\right\}
$$

are $\eta^{\prime}$-regular $\delta$-fine partitions in $A$ anchored in $E$ [12, Lemma 1.2]. Thus

$$
\begin{aligned}
\sum_{i=1}^{q}\left|F\left(B_{i}\right)\right| & =\sum_{x_{i} \in \mathrm{cl}^{*} B}\left|G\left(B_{i} \cap B\right)\right|+\sum_{x_{i} \notin \mathrm{cl}^{*} B}\left|G\left(B_{i}-\left[B_{i}-B\right]\right)\right| \\
& <\alpha+\sum_{i=1}^{q}\left|G\left(B_{i}\right)\right|+\sum_{x_{i} \notin \mathrm{Cl}^{*} B}\left|G\left(B_{i}-B\right)\right|<3 \alpha
\end{aligned}
$$

and applying again Remark 2.3, we see that $V_{*} F(E) \leq \alpha$. It follows that $F$ is a simple charge in $A$. Using Theorem 2.11, we conclude $F^{\prime} \in \mathcal{R}_{s}(A ; \lambda)$ and

$$
G(A \cap B)=F(A)=\int_{A} F^{\prime} d \lambda .
$$

Moreover, $F^{\prime}(x)=G^{\prime}(x)$ for almost all $x \in B$ by Remark 2.10. Applying the previous argument to $H=G \mathrm{~L}(A-B)$, we obtain $H^{\prime}(x)=G^{\prime}(x)$ for almost all $x \in A-B$. Since $G=F+H$ yields $G^{\prime}(x)=F^{\prime}(x)+H^{\prime}(x)$ whenever the derivates exist, $F^{\prime}=f G^{\prime}$ almost everywhere in $A$. Now equation (3.4) together with Theorem 2.11 and Proposition 2.9 imply $f \in \mathcal{R}_{s}(A ; G)$ and

$$
\hat{G}\left[\Sigma\left(f^{+}, A\right)\right]=G(A \cap B)=\int_{A} f G^{\prime} d \lambda=\int_{A} f d G .
$$

As $\hat{G}\left[\Sigma\left(f^{-}, A\right)\right]=0$, the proof is completed.

Corollary 3.7 Let $A \in B V_{c}$ be a union of $B V$ sets $B$ and $C$, and let $f$ be $a$ function on $\mathrm{cl}^{*} A$ with $f \in \mathcal{R}_{s}(B ; \lambda) \cap \mathcal{R}_{s}(C ; \lambda)$. If $f \notin \mathcal{R}_{s}(A ; \lambda)$, then either $f \uparrow \mathrm{cl}^{*} B$ or $f \uparrow \mathrm{cl}^{*} C$ has no s-integrable extension to $\mathrm{cl}^{*} A$. 
Proof. We may assume that the sets $B$ and $C$ are disjoint. Suppose both $f\left\lceil\mathrm{cl}^{*} B\right.$ and $f\left\lceil\mathrm{cl}^{*} C\right.$ have $s$-integrable extensions to $\mathrm{cl}^{*} A$ denoted by $f_{B}$ and $f_{C}$, respectively. Then $f=\chi_{B} f_{B}+\chi_{C} f_{C}$ almost everywhere in $\operatorname{cl}^{*} A$, and a contradiction follows from Propositions 3.6 and 2.4.

Remark 3.8 A function $f$ satisfying the assumption of Corollary 3.7 has been constructed by Z. Buczolich [11, Example 5.21].

Question 3.9 Let $G$ be a charge in $A \in B V_{c}$.

1. Is $\mathcal{B V}^{\infty}\left(\mathrm{cl}^{*} A\right)$ a subfamily of $\mathcal{R}_{s}(A ; G)$ or $\mathcal{R}(A ; G)$ ?

2. If $G$ is a regular charge, does the equality

$$
\int_{A} f d G=\hat{G}\left[\Sigma\left(f^{+}, A\right)\right]-\hat{G}\left[\Sigma\left(f^{-}, A\right)\right] .
$$

hold for each essentially bounded $B V$ function in $\mathcal{R}(A ; G)$ or $\mathcal{R}_{s}(A ; G)$ ?

The following comments about Question 3.9 are in place.

1. For $m=1$ and a primitive charge $G$, the first question was answered affirmatively in [1], and by a different method in [4, Section 5].

2. Example 2.2 shows that without the assumption of regularity the answer to the second question is negative.

\section{Linear Functionals on $\mathcal{B V}_{c}^{\infty}(\Omega)$}

Let $\Omega \subset \mathbb{R}^{m}$ be an open set, and let $G$ be a charge in $\Omega$. For $\varphi \in C_{c}^{1}(\Omega)$, let

$$
\int_{\Omega} \varphi d G=\int_{A} \varphi d G
$$

where $A \in B V_{c}(\Omega)$ contains $\operatorname{supp} \varphi$. It follows from Proposition 2.8 that the definition of $\int_{\Omega} \varphi d G$ does not depend on the choice of $A$.

Theorem 4.1 Let $G$ be a charge in an open set $\Omega \subset \mathbb{R}^{m}$. The map $\varphi \mapsto$ $\int_{\Omega} \varphi d G$ on $C_{c}^{1}(\Omega)$ has a unique extension to a continuous linear functional $\Lambda_{G}$ on $\mathcal{B V}_{c}^{\infty}(\Omega)$ given by $\Lambda_{G}(f)=\hat{G}\left[\Sigma\left(f^{+}, \Omega\right)\right]-\hat{G}\left[\Sigma\left(f^{-}, \Omega\right)\right]$. If $\int_{\Omega} \varphi d G=0$ for each $\varphi \in C_{c}^{1}(\Omega)$, then $G(A)=0$ for every $A \in B V_{c}(\Omega)$. 
Proof. If a sequence $\left\{f_{n}\right\}$ in $\mathcal{B V}_{c}^{\infty}(\Omega) \tau$-converges to an $f \in \mathcal{B V}_{c}^{\infty}(\Omega)$, then the sequences $\left\{\Sigma\left(f_{n}^{ \pm}, \Omega\right)\right\}$ in $B V_{c}(\Omega \times \mathbb{R}) \tau$-converge to $\Sigma\left(f^{ \pm}, \Omega\right) \in B V_{c}(\Omega \times \mathbb{R})$. It follows from Lemma 3.2 that the map $\Lambda_{G}: \mathcal{B} \mathcal{V}_{c}^{\infty}(\Omega) \rightarrow \mathbb{R}$ is continuous. As $\Lambda_{G}(\varphi)=\int_{\Omega} \varphi d G$ for each $\varphi \in C_{c}^{1}(\Omega)$ (Theorem 3.4), the functional $\Lambda_{G}$ is linear on $C_{c}^{\infty}(\Omega)$. This completes the proof, since $C_{c}^{\infty}(\Omega)$ is a dense subspace of $\mathcal{B V}_{c}^{\infty}(\Omega)$ and $G(A)=\Lambda_{G}\left(\chi_{A}\right)$ for every $A \in B V_{c}(\Omega)$.

Proposition 4.2 Let $G$ and $G_{1}, G_{2}, \ldots$ be charges in an open set $\Omega \subset \mathbb{R}^{m}$. If the sequence $\left\{G_{n}\right\}$ converges to $G$ monotonically or uniformly, then $\Lambda_{G}(f)=$ $\lim \Lambda_{G_{n}}(f)$ for each $f \in \mathcal{B V}_{c}^{\infty}(\Omega)$. Moreover, if $\left\{G_{n}\right\}$ converges uniformly, then $\left\{\Lambda_{G_{n}}\right\}$ converges uniformly on every family $\mathcal{E} \subset \mathcal{B V}_{c}^{\infty}(\Omega)$ with $\sup \left\{\|f\|_{\infty}: f \in \mathcal{E}\right\}<+\infty$.

Proof. If $C \in B V_{c}(\Omega \times \mathbb{R})$, then $C^{t}=\emptyset$ whenever $|t|$ is sufficiently large. Thus $\hat{G}(C)=\lim \widehat{G_{n}}(C)$ by Lemma 3.2 and the monotone or dominated convergence theorem. Moreover, if $\left\{G_{n}\right\}$ converges uniformly, then $\left\{\hat{G}_{n}\right\}$ converges uniformly on each family $B V_{c}(\Omega \times(a, b))$ where $a<b$ are real numbers. The proposition follows.

For an open set $\Omega \subset \mathbb{R}^{m}$, we denote by $\mathcal{B} \mathcal{V}_{\text {loc }}(\Omega)$ the linear space of all locally $B V$ functions in $\Omega\left[6\right.$, Section 5.1]. If $f$ belongs to $\mathcal{B} \mathcal{V}_{\text {loc }}(\Omega)$, or to the Sobolev space $W_{\text {loc }}^{1,1}(\Omega)\left[6\right.$, Section 4.1], we give $D f=\left(D_{1} f, \ldots, D_{m} f\right)$ the appropriate meaning.

Let $\Omega \subset \mathbb{R}^{m}$ be an open set. By $W_{\text {loc }}(\Omega)$ we denote the linear space of all functions $f \in L_{\text {loc }}^{1}(\Omega ; \lambda)$ whose distributional derivatives are charges in $\Omega$. More precisely, $f \in W_{\text {loc }}(\Omega)$ if and only if $f \in L_{\text {loc }}^{1}(\Omega ; \lambda)$ and there are charges $D_{1} f, \ldots, D_{m} f$ in $\Omega$ such that $\int_{\Omega} f \operatorname{div} v d \lambda=-\sum_{i=1}^{m} \int_{\Omega} v_{i} d\left(D_{i} f\right)$ for each vector field $v=\left(v_{1}, \ldots, v_{m}\right)$ in $C_{c}^{1}\left(\Omega, \mathbb{R}^{m}\right)$. As usual, we let $D f=$ $\left(D_{1} f, \ldots, D_{m} f\right)$, and denote $\sum_{i=1}^{m} \int_{\Omega} v_{i} d\left(D_{i} f\right)$ by $\int_{\Omega} v \cdot d(D f)$. In view of Theorem 4.1, the distributional gradient $D f$ of $f \in W_{\text {loc }}(\Omega)$ is determined uniquely, and it follows from Theorem 2.17 that

$$
\int_{\Omega} v \cdot d(D f)=\int_{\Omega} v \cdot D f d \lambda
$$

whenever $v \in C_{c}^{1}\left(\Omega, \mathbb{R}^{m}\right)$ and $f$ is a function almost differentiable in $\Omega$. In particular, for each function $f$ almost differentiable in $\Omega$, the distributional derivatives $D_{i} f$ are the indefinite integrals in $\Omega$ with respect to $\lambda$ of the classical partial derivatives $\partial f / \partial x_{i}$ defined almost everywhere in $\Omega$ [14, Stepanoff's Theorem 6.6.8]. 
Proposition 4.3 If $\Omega \subset \mathbb{R}^{m}$ is an open set, then

$$
W_{\text {loc }}^{1,1}(\Omega)=\mathcal{B V}_{\text {loc }}(\Omega) \cap W_{\text {loc }}(\Omega) .
$$

Moreover, a function $f \in W_{\mathrm{loc}}(\Omega)$ belongs to $W_{\mathrm{loc}}^{1,1}(\Omega)$ if and only if the measure $V_{*}\left(D_{i} f\right)$ in $\Omega$ is absolutely continuous and locally finite.

Proof. As the other inclusion is obvious, assume $f \in \mathcal{B V}_{\text {loc }}(\Omega) \cap W_{\text {loc }}(\Omega)$. For $i=1, \ldots, m$, the locally finite signed measure $D_{i} f$ in $\Omega$ is absolutely continuous, since it is also a charge in $\Omega$. Thus the density of $D_{i} f$ belongs to $L_{\text {loc }}^{1}(\Omega ; \lambda)$ which means $f \in W_{\text {loc }}^{1,1}(\Omega)$. The rest of the proposition is an immediate consequence of [3, Proposition 5.9].

If $\Omega \subset \mathbb{R}^{m}$ is an open set and $v=\left(v_{1}, \ldots, v_{m}\right)$ is a vector field in the space $W_{\text {loc }}\left(\Omega, \mathbb{R}^{m}\right)$, we let $\operatorname{div} v=\sum_{i=1}^{m} D_{i} v_{i}$.

Proposition 4.4 Let $\Omega \subset \mathbb{R}^{m}$ be an open set, and let $v: \Omega \rightarrow \mathbb{R}^{m}$ be a continuous vector field. If $v \in W_{\mathrm{loc}}\left(\Omega, \mathbb{R}^{m}\right)$, then $\operatorname{div} v(A)=\int_{\partial^{*} A} v \cdot \nu_{A} d \mathcal{H}$ for each $A \in B V_{c}(\Omega)$.

Proof. Let $v=\left(v_{1}, \ldots, v_{m}\right)$ and select an $A \in B V_{c}(\Omega)$. By [6, Section5.2.2], there is a sequence $\left\{\varphi_{n}\right\}$ in $C_{c}^{1}(\Omega)$ converging to $\chi_{A}$ in $\mathcal{B} \mathcal{V}_{c}^{\infty}(\Omega)$ and such that $\left\{D \varphi_{n}\right\}$ converges weakly to $D \chi_{A}$. Applying Proposition 2.4 and Theorem 4.1, we obtain

$$
\begin{aligned}
\operatorname{div} v(A) & =\sum_{i=1}^{m} \Lambda_{D_{i} v_{i}}\left(\chi_{A}\right)=\lim _{n \rightarrow \infty} \sum_{i=1}^{m} \Lambda_{D_{i} v_{i}}\left(\varphi_{n}\right)=\lim _{n \rightarrow \infty} \sum_{i=1}^{m} \int_{\Omega} \varphi_{n} d\left(D_{i} v_{i}\right) \\
& =-\lim _{n \rightarrow \infty} \int_{\Omega} v \cdot D \varphi_{n} d \lambda=-\int_{\Omega} v \cdot d\left(D \chi_{A}\right) \\
& =\int_{\partial^{*} A} v \cdot \nu_{A} d \mathcal{H} . *
\end{aligned}
$$

\section{References}

[1] B. Bongiorno and V. Skvortsov, Multipliers for some generalized Riemann integrals in the real line, Real Anal. Ex., 20 (1994-95), 212-218.

[2] B. Bongiorno, L. Di Piazza and V. Skvortsov, A new full descriptive characterization of Denjoy-Perron integral, to appear.

[3] Z. Buczolich and W. F. Pfeffer, Variations of additive functions, to appear. 
[4] T. De Pauw, Topologies for the space of $B V$-integrable functions in $\mathbb{R}^{N}$, to appear.

[5] R. Engelking, General Topology, PWN, Warsaw, 1977.

[6] L. C. Evans and R. F. Gariepy, Measure Theory and Fine Properties of Functions, CRC Press, Boca Raton, 1992.

[7] H. Federer, Geometric Measure Theory, Springer-Verlag, New York, 1969.

[8] J. Mařík, The surface integral, Czechoslovak Math. J., 6 (1956), 522-558.

[9] U. Massari and M. Miranda, Minimal Surfaces in Codimension One, North-Holland, Amsterdam, 1984.

[10] J. W. Mortensen and W. F. Pfeffer, Multipliers for the generalized Riemann integral, J. Math. Anal. Appl., 187 (1994), 538-547.

[11] W. F. Pfeffer, The Gauss-Green theorem, Adv. Math., 87 (1991), 93-147.

[12] W. F. Pfeffer, A descriptive definition of a variational integral and applications, Indiana Univ. Math. J., 40 (1991), 259-270.

[13] W. F. Pfeffer, An integral in geometric measure theory, Atti Sem. Mat. Fis. Univ. Modena, 41 (1993), 59-76.

[14] W. F. Pfeffer, The Riemann Approach to Integration, Cambridge Univ. Press, Cambridge, 1993.

[15] W. F. Pfeffer On variations of one-dimensional charges, to appear.

[16] W. Rudin, Real and Complex Analysis, McGraw-Hill, New York, 1987.

[17] W. Rudin, Functional Analysis, McGraw-Hill, New York, 1991.

[18] S. Saks, Theory of the Integral, Dover, New York, 1964.

[19] W. L. C. Sargent, On the integrability of products, J. London Math. Soc., 23 (1948), 28-34.

[20] E. M. Stein, Singular Integrals and Differentiability Properties of Function, Princeton Univ. Press, Princeton, 1970.

[21] B. S. Thomson, Derivates of Interval functions, Mem. Amer. Math. Soc., 452, Providence, 1991.

[22] A. I. Volpert, The spaces BV and quasilinear equations, Math. USSR-SB., 2 (1967), 255-267. 